\title{
Surface perturbations of a shallow viscous fluid heated from below and the $(2+1)$-dimensional Burgers equation
}

\author{
R. A. Kraenkel and J. G. Pereira \\ Instituto de Física Teórica, Universidade Estadual Paulista, Rua Pamplona 145, 01405 São Paulo, São Paulo, Brazil \\ M. A. Manna \\ Laboratoire de Physique Mathématique, Université des Sciences et Tecniques du Languedoc, 34060 Montpellier CEDEX, France
}

(Received 6 March 1991)

\begin{abstract}
The $(2+1)$-dimensional Burgers equation is obtained as the equation of motion governing the surface perturbations of a shallow viscous fluid heated from below, provided the Rayleigh number of the system satifies the condition $R \neq 30$. A solution to this equation is explicitly exhibited and it is argued that it describes the nonlinear evolution of a nearly one-dimensional kink.
\end{abstract}

PACS number(s): 47.20.Bp, 47.35. $+\mathrm{i}$

The system formed by a fluid heated from below, the so-called Bénard problem, has been a standard model for many studies in fluid dynamics [1]. In most cases, however, the main interest has been concentrated in the convection phenomena. While considering the same system, our concern here will be quite different, since we will be interested in the study of surface waves in a shallow fluid, and in situations for which the Rayleigh number $(R)$ is well below that determined by the onset of convection. Furthermore, we shall only consider systems for which the upper boundary is a two-dimensional surface. It is important to remark that the shallow-fluid approximation implies that the depth of the fluid is much smaller than the wavelength of the surface waves. However, since we shall restrict ourselves to the study of long surface waves, on whose description the slow variables [2] play a very important role, the depth of the fluid will not necessarily be small. As a matter of fact, we shall assume later a deep enough fluid such that surface-tension effects can be safely disregarded. We should still add that the heat flux from below is essential to our problem only because we are interested in studying the constraints that the thermal boundary conditions impose on the system.

By using the concept of a weak coordinate [3], it has recently been shown [4] that nearly one-dimensional undamped waves described by the Kadomtsev-Petviashvili equation may propagate in a shallow viscous fluid, provided the Rayleigh number of the system satisfies the condition $R=30$. These solitary waves were sustained by the adverse temperature gradient. In other words, they are possible because at the point $R=30$, the amount of energy released by buoyancy exactly compensates the amount dissipated by viscosity [5].

Following those developments, we shall show here that, considering a system for which the upper boundary is a two-dimensional surface with the $\mathrm{x}$-transverse coordinate $y$ assumed to be weak, and using an appropriate scaling in the definition of the slow variables, a $(2+1)$ dimensional Burgers equation [6] is obtained as the equation of motion of surface perturbations, provided $R \neq 30$. This equation admits a progressive wave solution, which will be explicitly exhibited, that describes a nearly onedimensional kink propagating in a viscous fluid. The approach we give here is valid for any Rayleigh number, and so it also includes the result discussed above that is valid strictly when $R=30$.

Let us then consider a Bénard system consisting of a fluid bounded below by a plane stress-free perfectly thermally conducting medium at $z=0$ and temperature $T=T_{b}$, and bounded above by a free surface which, when at rest, lies at $z=d$. The depth $d$ of the fluid is such that the buoyancy effect is predominant when compared to the influence of the surface-tension variation with temperature. This is the reason we will assume a vanishing surface tension. The equations governing the hydrodynamical flow of a viscous fluid can be simplified considerably by using the Boussinesq approximation. The origin of this simplification is the smallness of the coefficient of thermal expansion $\gamma$. As for most situations of practical occurrence $\gamma$ is indeed small, ranging usually from $10^{-3}$ to $10^{-4}$, this approximation does not impose severe restrictions from the physical point of view. In this approximation, the equations that describe the motion of a fluid are given by

$$
\begin{aligned}
& \nabla \cdot \mathbf{v}=0, \\
& \rho_{0} \frac{d \mathbf{v}}{d t}=-\nabla p+\mu \nabla^{2} \mathbf{v}+\mathbf{g} \rho, \\
& \frac{d T}{d t}=\kappa \nabla^{2} T, \\
& \rho=\rho_{0}\left[1-\gamma\left(T-T_{0}\right)\right],
\end{aligned}
$$

where $d / d t=\partial / \partial t+\mathbf{v} \cdot \nabla$ is the convective derivative, $\mathbf{v}=(u, v, w)$ is the fluid velocity, and $p$ is the pressure. The viscosity $\mu$, thermal diffusivity $\kappa$, and coefficient of thermal expansion $\gamma$, are constant. $T_{0}$ and $\rho_{0}$ are the reference temperature and density, respectively.

On the upper free surface $z=d+\eta(x, y, t)$, the boundary conditions are [7]

$\eta_{t}+u \eta_{x}+v \eta_{y}=w$, 
$\left(p-p_{a}\right) \eta_{x}-\mu\left[2 u_{x} \eta_{x}-\left(u_{z}+w_{x}\right)+\left(u_{y}+v_{x}\right) \eta_{y}\right]=0$,

$p-p_{a}+\mu\left[\left(w_{x}+u_{z}\right) \eta_{x}-2 w_{z}+\left(w_{y}+v_{z}\right) \eta_{y}\right]=0$,

$\left(p-p_{a}\right) \eta_{y}-\mu\left[\left(v_{x}+u_{y}\right) \eta_{x}-\left(v_{z}+w_{y}\right)+2 v_{y} \eta_{y}\right]=0$,

and

$$
\widehat{\mathbf{n}} \cdot \nabla T=-\frac{F}{k},
$$

where $\hat{\mathbf{n}}$ is the unit vector normal to the free surface, given by

$$
\widehat{\mathbf{n}}=\left(-\eta_{x},-\eta_{y}, 1\right) / N, \quad N=\left(1+\eta_{x}^{2}+\eta_{y}^{2}\right)^{1 / 2} .
$$

$F$ is normal heat flux, $k$ is the thermal conductivity, and $p_{a}$ is a constant pressure exerted on the upper free surface.

An important point is the dynamical boundary condition that is to be satisfied at the lower plane. We suppose that the sliding resistance between two portions of the fluid is much greater than between the fluid and the plane [8]. Under this condition, it is reasonable to assume a stress-free lower surface, which implies [1]

$$
w=u_{z}=v_{z}=0
$$

for $z=0$.

The static solution to these equations depends only on the coordinate $z$ and is given by

$$
\begin{aligned}
& T_{s}=T_{0}-\frac{F}{k}(z-d), \quad \rho_{s}=\rho_{0}\left[1+\frac{\gamma F}{k}(z-d)\right], \\
& p_{s}=p_{a}-g \rho_{0}\left[(z-d)+\frac{\gamma F}{2 k}(z-d)^{2}\right) .
\end{aligned}
$$

In order to get the dimensionless form of the equations, boundary conditions, and static solutions, we adopt $d$ as the unit of length, $d^{2} / \kappa$ as the unit of time, $\rho_{0} d^{3}$ as the unit of mass, and $F d / k$ as the unit of temperature. Furthermore, we introduce three dimensionless parameters: the Prandtl number $\sigma=\mu / \rho_{0} \kappa$, the Rayleigh number $R=\rho_{0} g \alpha F d^{4} / k \kappa \mu$, and the Galileo number $G=g d^{3} \rho_{0}^{2} / \mu^{2}$.

Now comes an important point: the definition of slow variables. We write [9]

$$
\xi=\epsilon^{\alpha}(x-c t), \quad \xi=\epsilon^{\alpha+1 / 2} y, \quad \tau=\epsilon^{\alpha+1} t,
$$

with $\epsilon$ a small positive parameter, and $\alpha$ a parameter that assumes either the value $\frac{1}{2}$ or 1 . The appropriate value for it will be discussed later. Considering now the expansions

$$
\begin{aligned}
& u=\epsilon\left(u_{0}+\epsilon u_{1}+\epsilon^{2} u_{2}+\cdots\right), \\
& v=\epsilon^{3 / 2}\left(v_{0}+\epsilon v_{1}+\epsilon^{2} v_{2}+\cdots\right), \\
& w=\epsilon^{\alpha+1}\left(w_{0}+\epsilon w_{1}+\epsilon^{2} w_{2}+\cdots\right), \\
& \eta=\epsilon\left(\eta_{0}+\epsilon^{1} \eta_{1}+\epsilon^{2} \eta_{2}+\cdots\right), \\
& p-p_{s}=\epsilon^{\alpha}\left(p_{0}+\epsilon p_{1}+\epsilon^{2} p_{2}+\cdots\right), \\
& T-T_{s}=\epsilon^{\alpha+1}\left(\theta_{0}+\epsilon \theta_{1}+\epsilon^{2} \theta_{2}+\cdots\right),
\end{aligned}
$$

where all quantities are dimensionless, we can obtain an order-by-order solution to the system of equations. Along this process, we shall take only integral powers of $\epsilon$. That this is really possible can be seen in the following way. When considering any integer order, we take every term containing powers of $\epsilon$ that may possibly contribute to that order. For example, to the first order will contribute, besides terms containing $\epsilon^{1}$ itself, terms containing $\epsilon^{\alpha}$ and $\epsilon^{\alpha+1}$. To the second order will contribute terms containing $\epsilon^{2}, \epsilon^{\alpha+1}, \epsilon^{2 \alpha}$, and $\epsilon^{2 \alpha+1}$, and so on. Consequently, depending on the value of $\alpha$, the first order will also include the order $\epsilon^{1 / 2}$, the second order will also include the order $\epsilon^{3 / 2}$, and so on. Thus, it is not necessary to consider separately the half-integer orders, since they have already been taken into account.

In the lowest order, the solution is given by

$$
\begin{aligned}
& u_{0}=f(\xi, \xi, \tau), \quad w_{0=}-z f_{\xi}(\xi, \zeta, \tau), \\
& \eta_{0}=\frac{1}{c} f(\xi, \zeta, \tau), \quad p_{0}=\epsilon^{1-\alpha} \frac{G \sigma^{2}}{c^{2}} f(\xi, \zeta, \tau),
\end{aligned}
$$

with $f(\xi, \zeta, \tau)$ an arbitrary function. In the next order we get

$$
\begin{aligned}
\theta_{0}= & \frac{1}{6}\left(z^{3}-3 z\right) f_{\xi}, \quad v_{0}=h(\xi, \zeta, \tau), \\
u_{1}= & g(\xi, \zeta, \tau)+\epsilon^{2 \alpha-1}\left[\frac{R}{720}\left(z^{6}-15 z^{4}+75 z^{2}\right)-\frac{3}{2} z^{2}\right] f_{\xi \xi} \\
& -\epsilon^{3 \alpha-1}\left[\frac{c}{24 \sigma}\left(z^{4}-6 z^{2}\right)\right] f_{\xi \xi \xi} \\
& +\epsilon^{\alpha}\left[G \sigma \eta_{1 \xi}+\frac{R}{c^{2}} f f_{\xi}\right) \frac{z^{2}}{2}, \\
w_{1}= & -z\left(g_{\xi}+h_{\xi}\right)-\epsilon^{2 \alpha-1} \\
& \times\left[\frac{R}{5040}\left(z^{7}-21 z^{5}+175 z^{3}\right)-\frac{z^{3}}{z}\right] f_{\xi \xi \xi} \\
& +\epsilon^{3 \alpha-1}\left[\frac{c}{120 \sigma}\left(z^{5}-10 z^{3}\right)\right] f_{\xi \xi \xi \xi} \\
& -\epsilon^{\alpha}\left(G \sigma \eta_{1 \xi \xi}+\frac{R}{c^{2}}\left(f_{\xi} f_{\xi}+f f_{\xi \xi}\right)\right] \frac{z^{3}}{6}, \\
p_{1}= & \left.\frac{R \sigma}{24}\left(z^{4}-6 z^{2}+5\right)-2 \sigma\right) f_{\xi} \\
& -\epsilon^{\alpha}\left(\frac{c}{2}\left(z^{2}-1\right)\right] f_{\xi \xi}+\epsilon^{1-\alpha}\left(G \sigma^{2} \eta_{1}+\frac{R \sigma}{2} \eta_{0}^{2}\right),
\end{aligned}
$$

with $g(\xi, \xi, \tau)$ and $h(\xi, \xi, \tau)$ arbitrary functions. The boundary condition on the upper free surface yields the equation

$$
\begin{aligned}
c^{2} \eta_{1 \xi}-c g_{\xi}= & f_{\tau}+2 f f_{\xi} \\
& +\epsilon^{2 \alpha-1}\left(\frac{31}{1008} c R-\frac{c}{2}\right) f_{\xi \xi \xi}+c h_{\xi} .
\end{aligned}
$$


At this order, there appears a solubility condition giving

$$
c^{2}=G \sigma^{2} \text {. }
$$

In the next order, we get

$$
\begin{gathered}
\theta_{1}=\epsilon^{\alpha-1}\left[\frac{c}{120}\left(z^{5}-10 z^{3}+25 z\right)\right] f_{\xi \xi}, \\
p_{2}=-\epsilon^{\alpha-1}\left[\frac{c R \sigma}{720}\left(z^{6}-15 z^{4}+75 z^{2}-61\right)\right. \\
\left.+\frac{c}{2}\left(z^{2}-1\right)\right] f_{\xi \xi} .
\end{gathered}
$$

These solutions and the remaining boundary conditions yield the equation

$$
\begin{aligned}
-\left(c^{2} \eta_{1 \xi}-c g_{\xi}\right)= & f_{\tau}+\left(1+\frac{R}{G \sigma}\right) f f_{\xi} \\
& -\epsilon^{\alpha-1} \sigma\left(4-\frac{2 R}{15}\right) f_{\xi \xi} \\
& +\epsilon^{2 \alpha-1} c\left(\frac{7}{6}+\frac{272}{5040} R \sigma-\frac{155}{5040} R\right) f_{\xi \xi \xi},
\end{aligned}
$$

as well as a relation between the arbitrary functions $f(\xi, \zeta, \tau)$ and $h(\xi, \zeta, \tau)$ :

$$
f_{\zeta}=h_{\xi} \text {. }
$$

The requirement of compatibility of Eqs. (12), (14), and (15) provides an evolution equation for $f$ :

$$
\begin{aligned}
& {\left[f_{\tau}+\left[\frac{3 G \sigma+R}{2 G \sigma}\right] f f_{\xi}-\epsilon^{\alpha-1} v f_{\xi \xi}\right.} \\
& \quad+\epsilon^{2 \alpha-1} c\left(\frac{1}{6}+\frac{17}{630} \sigma R\right) f_{\xi \xi \xi} \\
& \quad=-\frac{c}{2} f_{\xi \xi},
\end{aligned}
$$

where

$$
v=2 \sigma-\frac{R \sigma}{15} .
$$

Suppose then we consider a system such that the Rayleigh number is in the region $R<30$, which means that the coefficient $v$ is positive. Taking $\alpha=1$ and neglecting the term proportional to $\epsilon$, Eq. (16) becomes

$$
\left[f_{\tau}+\left[\frac{3 G \sigma+R}{2 G \sigma}\right] f f_{\xi}-v f_{\xi \xi}\right]_{\xi}=-\frac{c}{2} f_{\xi \xi} .
$$

By transforming $f$ according to

$$
f \rightarrow \frac{2 G \sigma}{3 G \sigma+R} f
$$

we obtain the equation

$$
\left(f_{\tau}+f f_{\xi}-v f_{\xi \xi}\right)_{\xi}=-\frac{c}{2} f_{\zeta \xi} .
$$

This is the $(2+1)$-dimensional Burgers equation [6]. In the limiting case of a one-dimensional upper surface, no $\zeta$ dependence is allowed for $f$ and it coincides with the usual $(1+1)$-dimensional Burgers equation. If no heat flux were present, the same equation would be obtained, but with

$$
v=2 \sigma \text {. }
$$
form

Equation (17) has a progressive wave solution of the

$$
f(\Lambda)=f(A \xi+B \xi-C \tau),
$$

whose explicit form is

$$
\begin{aligned}
f(\Lambda)= & \frac{(3 G \sigma+R)\left(2 C-c B^{2}\right)}{4 G \sigma} \\
& \times\left[1-\tanh \left[\frac{\left(2 C-c B^{2}\right)}{4 v}\left(\Lambda-\Lambda_{0}\right)\right],\right.
\end{aligned}
$$

where $A, B$, and $C$ are constants depending on the initial conditions. It represents a nearly one-dimensional kink, which can be considered as a kind of bore [10]. The condition $v>0$ reflects the fact that the system is predominantly dissipative, i.e., the amount of energy released by buoyancy is smaller than the amount dissipated by viscosity.

Suppose now we slowly increase the heat flux. As the Rayleigh number approaches the point $R=30$, the coefficient $v$ approaches zero and the system becomes less and less dissipative. At the point $R=30, v$ vanishes, indicating that the amount of energy dissipated by viscosity is exactly compensated by the amount released by buoyancy. At this point, therefore, dissipation does not play its role and the system becomes predominantly dispersive. In fact, putting $v=0$, taking $\alpha=\frac{1}{2}$, and rescaling conveniently $\xi, \xi$, and $f$, Eq. (16) becomes [4]

$$
\left(f_{\tau}+6 f f_{\xi}+f_{\xi \xi \xi}\right)_{\xi}=-3 f_{\zeta \xi},
$$

which is the widely studied integrable KadomtsevPetviashvili equation.

If we continue to increase the heat flux more and more, the Rayleigh number of system will reach the region $R>30$, and consequently $v$ will become negative. The system will then be antidissipative, which means that the amount of energy released by buoyancy will be greater than the amount dissipated by viscosity. In this case, the surface perturbations will again be governed by the $(2+1)$-dimensional Burgers equation, and the solution given be Eq. (19) will represent a nearly one-dimensional antikink propagating in the opposite direction. It should be noticed, however, that this solution is not valid for an arbitrarily large $R$, since in this region other phenomena, which are not considered by our approach, may take place.

It has now become clear why we have considered both cases, $\alpha=1$ and $\alpha=\frac{1}{2}$. When $\nu \neq 0$, the term containing the second derivative in Eq. (16) is of lower order than the term containing the third derivative, and by setting $\alpha=1$ it gives a true contribution to the equation. When $v=0$, however, we are left with only the third-derivative term, which will contribute when $\alpha=\frac{1}{2}$.

Finally, it is interesting to remark that the Hopf-Cole transformation [11] 


$$
f=-2 v \frac{\partial}{\partial \xi} \ln \phi,
$$

which transforms the ordinary Burgers equation into the heat equation, in this case leads Eq. (18) to the nonlinear equation

$$
\left[\frac{\phi_{\tau}-v \phi_{\xi \xi}}{\phi}\right]_{\xi}=-\frac{c}{2}(\ln \phi)_{\zeta \xi} .
$$

That the Hopf-Cole transformation does not lead to the $(2+1)$-dimensional heat equation is not surprising, since, while the heat equation would put $\xi$ and $\zeta$ in the same footing, Eq. (18) considers $\zeta$ as a weak coordinate.

In conclusion, we have obtained in this paper a $(2+1)$ - dimensional Burgers equation as the equation governing the upper-surface perturbations of a shallow viscous fluid heated from below, provided $R \neq 30$. A progressive-wave solution to this equation has been explicitly exhibited, which represents a nearly one-dimensional kink. The critical Rayleigh number $R=30$ is the point where dissipation is completely compensated by the buoyancy effect generated by the heat flux. At this point the system becomes dispersive and the equation governing the surface perturbations turns out to be the Kadomtsev-Petviashvili equation.

The authors would like to thank Conselho Nacional de Desenvolvimento Científico e Tecnológico (CNPq), Brazil, for partial financial support.
[1] S. Chandrasekhar, Hydrodynamic and Hydromagnetic Stability (Clarendon, Oxford, 1955).

[2] R. K. Dodd, J. C. Eilbeck, J. D. Gibbon, and H. C. Morris, Solitons and Nonlinear Wave Equations (Academic, London, 1982).

[3] B. B. Kadomtsev and V. I. Petviashvili, Dokl. Akad. Nauk SSSR 192, 753 (1970) [Sov. Phys. Dokl. 15, 539 (1970)].

[4] S. M. Kurcbart et al., Phys. Lett. A 148, 53 (1990).

[5] C. M. Alfaro and M. C. Depassier, Phys. Rev. Lett. 62, 2597 (1989).

[6] M. Bartuccelli, P. Pantano, and T. Brugarino, Lett. Nuovo
Cimento 37, 433 (1983).

[7] J. V. Wehausen and E. V. Laitone, in Encyclopaedia of Physics, edited by S. Flügge (Springer, Berlin, 1960), Vol. 9.

[8] G. G. Stokes, Trans. Cambridge Philos. Soc. 8, 287 (1845) [reprinted in Mathematical and Physical Papers (Johnson Reprint Corporation, New York, 1966), Vol. 1, p. 75].

[9] C. H. Su and C. S. Gardner, J. Math. Phys. 10, 536 (1969).

[10] J. J. Stoker, Water Waves (Interscience, London, 1982).

[11] G. B. Whitham, Linear and Nonlinear Waves (Wiley, New York, 1974). 